\title{
A Stochastic Dynamic Programming Model for Hydropower Scheduling with State-dependent Maximum Discharge Constraints
}

This paper was downloaded from TechRxiv (https://www.techrxiv.org).

\section{LICENSE}

CC BY 4.0

SUBMISSION DATE / POSTED DATE

$10-08-2021 / 12-08-2021$

\section{CITATION}

Schäffer, Linn; Helseth, Arild; Korpås, Magnus (2021): A Stochastic Dynamic Programming Model for Hydropower Scheduling with State-dependent Maximum Discharge Constraints. TechRxiv. Preprint. https://doi.org/10.36227/techrxiv.15141885.v1

DOI 


\title{
A Stochastic Dynamic Programming Model for Hydropower Scheduling with State-dependent Maximum Discharge Constraints
}

\author{
Linn Emelie Schäffer, Arild Helseth, Senior Member, IEEE, and Magnus Korpås, Member, IEEE
}

\begin{abstract}
We present a medium-term hydropower scheduling model that includes state-dependent environmental constraints on maximum discharge. A stochastic dynamic programming algorithm is used to enable modelling of nonconvex relationships in the problem formulation. The model is applied in a case study of a Norwegian hydropower system with multiple reservoirs. We find that the maximum discharge constraint significantly impacts the water values and simulated operation of the hydropower system. A main finding is that the nonconvex characteristics of the environmental constraint is reflected in the water values, implying a nonconvex objective function. Operation according to the computed water values is simulated for cases with and without the environmental constraint. Even though operation of the system changes considerably when the environmental constraint is included, the total electricity generation over the year is kept constant, and the total loss in expected profit limited to less than $0.8 \%$.
\end{abstract}

Index Terms-Environmental factors, Hydroelectric power generation, Optimization methods, Power generation scheduling, Stochastic processes.

\section{NOMENCLATURE}

Index Sets

$\mathcal{D}_{h} \quad$ Set of discharge segments per hydropower plant $h$

$\mathcal{G} \quad$ Set of subsets used in the triangle method

$\mathcal{H} \quad$ Set of hydropower plants

$\mathcal{H}_{h}^{u p} \quad$ Set of hydropower plants with outlet to plant $h$

$\mathcal{J} \quad$ Set of iterations in SDP algorithm

$\mathcal{K} \quad$ Set of time steps within each stage

$\mathcal{N} \quad$ Set of reservoir segments per reservoir

$\mathcal{N}_{g}^{\text {Diag }} \quad$ Set of $\gamma$ for each diagonal $g$ used in the triangle method

$\mathcal{S}^{p} \quad$ Set of endogenous states

$\mathcal{S}^{u} \quad$ Set of stochastic states

$\mathcal{T} \quad$ Set of stages

\section{Decision Variables}

$\alpha_{t+1} \quad$ Expected future profit in stage $t$, in $\frac{€}{M W h}$

$\beta_{h, n} \quad$ Auxiliary variable for segment $n$ in reservoir $h$

$\chi_{g} \quad$ Auxiliary variable for set $g$

$\gamma_{n, m} \quad$ Weighting variable for reservoir segments $n, m$

$f_{k, h} \quad$ Spillage in time step $k$ from reservoir $h$, in $m^{3} / s$

$p_{k, h} \quad$ Generated electricity in time step $k$ from hydropower station $h$, in $M W$

This work was supported by the Research Council of Norway, Project nr. 257588. (Corresponding author: Linn Emelie Schäffer.) L. E. Schäffer and Magnus Korpås are with the Department of Electric Power Engineering, A. Helseth is with SINTEF Energy Research, Trondheim 7034, Norway (e-mail: arild.helseth@sintef.no). $q_{k, h, d} \quad$ Discharge in time step $k$ per segment $d$ from reservoir $h$, in $\mathrm{m}^{3} / \mathrm{s}$

$s_{k, h}^{r e s} \quad$ Slack variable used to penalise low storage volumes in time step $k$ in the reservoir $h$, in $M m^{3}$

$v_{k, h} \quad$ Storage volume in reservoir $h$, time step $k, \mathrm{Mm}^{3}$ Parameters

$\Delta \quad$ Change in water value matrix

$\eta_{h, d} \quad$ Efficiency per hydropower plant $h$ and discharge segment $d, \frac{M W}{m^{3} / s}$

$\hat{H} \quad$ Hydropower plant restricted by the state-dependent maximum discharge constraint

$\hat{Z}_{t} \quad$ Sum inflow per stage $t$, in $\mathrm{Mm}^{3}$

$\lambda_{t} \quad$ Power price in stage $t$, in $\frac{€}{M W h}$

$\omega_{h} \quad$ Scaling factor, scaling inflow to each reservoir $h$

$\phi_{k} \quad$ Distribution factor of inflow to each time step $k$

$\Phi_{j, t}(\ldots) \quad$ Expected future profit matrix, in stage $t$, iteration j

$\Psi_{j, t}^{h}(\ldots) \quad$ Water value matrix for reservoir $h$, stage $t$, iteration $j$

$\theta_{k} \quad$ Scaling factor for price variability in time stage $k$

$\xi_{t} \quad$ Environmental state in stage $t$

$C^{R} \quad$ Penalty cost for low reservoir filling, in $\frac{€}{m^{3} / \mathrm{s}}$

$C^{S} \quad$ Penalty cost for spillage, in $\frac{€}{\mathrm{~m}^{3} / \mathrm{s}}$

$F^{H} \quad$ Conversion factor, number of hours in time step

$F^{C} \quad$ Conversion factor, flow to volume, $\frac{\mathrm{Mm}^{3}}{\mathrm{~m}^{3} / \mathrm{s}}$

$F V_{n, m} \quad$ Expected future profit for reservoir segments $n$ and $m$, in $€$

$J \quad$ Maximum number of iterations in SDP algorithm

$K \quad$ Number of time steps in each stage

$\operatorname{Pr}(\ldots) \quad$ Transition probability matrix

$Q_{h}^{\min } \quad$ Minimum discharge limit of hydropower plant $h$, in $\mathrm{m}^{3} / \mathrm{s}$

$Q_{h, d}^{\max } \quad$ Maximum discharge per reservoir $h$ and discharge segment, in $\mathrm{m}^{3} / \mathrm{s}$

$s^{p} \quad$ Endogenous state

$s_{t}^{u} \quad$ Stochastic state in stage $t$

$T \quad$ Number of stages in the planning horizon

$t_{A-E} \quad$ Specific time markers used in the definition of the environmental constraints

$V_{h}^{\text {lim }} \quad$ Environmental threshold for reservoir $h$, in $\mathrm{Mm}^{3}$

$V_{n, h}^{\text {seg }} \quad$ Volume of each segment $n$ in reservoir $h$, in $\mathrm{Mm}^{3}$

$V_{h}$ Initial storage volume in reservoir $h$, in $\mathrm{Mm}^{3}$

$V_{h}^{\max } \quad$ Maximum storage volume in reservoir $h$, in $\mathrm{Mm}^{3}$

$V_{h}^{\text {min }} \quad$ Minimum storage volume in reservoir $h$, in $\mathrm{Mm}^{3}$ 
$Z_{h} \quad$ Inflow to reservoir $h$, in $\mathrm{Mm}^{3}$

\section{INTRODUCTION}

$\mathbf{T}$ HROUGH the European Green Deal, the EU has set ambitious targets for both climate change mitigation and broader environmental sustainability [1]. To align capital flows with these policy goals, the EU is in the process of defining requirements for environmentally sustainable activities. Hence, power producers have strong incentives to operate in an environmentally sustainable way.

Like all power plants, hydropower operations may modify the surrounding ecosystems [2]. Environmental concerns of hydropower operations are often related to alteration of the flow regime downstream the plant [3], but can also be related to the changes in in water temperatures or changed water volumes in the reservoirs. To protect ecological and recreational interests, regulation often imposes mitigation measures and limitations on operation, see e.g. [4] and [5].

Environmental regulation should be incorporated as constraints in optimization-based hydropower scheduling models. Omitting such constraints can result in misestimation of hydropower electricity generation, revenues, and the amount of flexibility hydrpower can provide to the electricity system [6]. The need for flexible resources is expected to increase as the transition of the European power system moves forward. In the transition towards a low-carbon system, hydropower can play an important role as a flexibility provider by responding to rapid fluctuations in intermittent renewable generation and load [7]. Additional constraints on operation may reduce this flexibility potential. To correctly represent hydropower operation requires properly accounting for environmental constraints imposed on hydropower production.

In this work we are concerned with operational hydropower scheduling, i.e. the sequence of decisions that are made leading up to the actual operation of the system. Due to the computational complexity, the scheduling problem is normally solved for different planning horizons and technical details. Mediumterm hydropower scheduling considers reservoir management under uncertainty over a planning horizon of several months up to a few years. In contrast, short-term scheduling usually concerns operational decisions over a period of days to weeks, and typically accounts for more technical details. In the decentralized Nordic system, medium-term scheduling models are used to compute water values, which are an essential input to the operational short-term models [8]. The purpose of water values is to reflect the long-term value of short-term operational decisions.

State-of-the-art methods for medium-term scheduling of hydropower systems use stochastic dual dynamic programming (SDDP) [9] algorithms. These algorithms solve the problem without discretising the state variables (such as reservoir volume and inflow), making it computationally tractable for systems with multiple reservoirs. For medium-term scheduling in the Nordic market, the combined SDP/SDDP method described in [10] is widely used. It combines SDDP with an outer layer based on stochastic dynamic programming (SDP) [11] to treat uncertainty in market prices. Still, a major drawback of the method is that non-convexities cannot be easily treated in the modelling.

Nonconvex problem formulations are typically needed to represent the complex interaction between power output and water [12], and unit commitment of generators [13]. The challenge of representing nonconvex relationships in the SDDP algorithm has frequently been addressed in the literature by the use of approximations, e.g [14]-[17]. A few studies also consider accurate modelling of non-convexities by the use of SDP [18] or stochastic dual dynamic integer programming (SDDiP), such as in [19] and [20].

SDP was used early on in hydropower planning, as it allows for explicit representation of uncertainty, e.g. in inflow and price [21]-[23]. The method has the advantage that it can represent nonconvex and nonlinear relationships. The main drawback of the method is that the state variables have to be discretised, causing the problem to grow exponentially in size with the number of state variables (e.g. reservoirs). The method is therefore best suited to solve systems with a small number of reservoirs, unless an aggregation technique is used like in [24] and [25]. In spite of this weakness, the SDP method is very well suited for accurate scheduling of hydropower systems with pronounced nonconvexities.

Regulators have imposed a wide range of environmental constraints on hydropower systems. Ecological flow requirements and maximum ramping rates are often applied, and may have significant impact on the flexibility of the hydro system. These constraints have been extensively studied in the technical literature, see e.g. [26]-[28], and can be included in hydropower scheduling models without compromising the convexity requirement of the SDDP algorithm. However, less research considers environmental constraints that involve statedependencies or logical conditions, which can not easily be treated in a convex model formulation [29]. Furthermore, only a very limited number of studies discuss the impact of environmental constraints on water values. To the best of our knowledge, the impact of environmental constraints on water values has only been discussed in [30] and [31]. In [31], an SDP-based model is used to evaluate the sensitivity of the water values to environmental flows and ramping restrictions. The authors of [30] find that incorporating the same environmental constraints into a linear programming based water value model has significant impacts on the profitability of hydropower plants with one or at most two turbines.

This work considers the representation of a particular type of state-dependent environmental constraint in the mediumterm scheduling of hydropower operation using an SDP algorithm. Our work differs from [30] and [31] in the type of constraints being considered. State-dependent maximum discharge constraints are imposed by the regulator on several Norwegian reservoirs, and will most likely be implemented in other hydropower reservoirs in the future. The purpose of the constraint is to retain inflow during spring, to meet the needs of ecological habitat and recreational use for high water levels in the reservoir through summer. The impact of volumedependent maximum discharge in medium-term scheduling has previously been studied in [20] using SDDiP. Compared to [20], we take a broader view of state-dependencies and 
study a version of the maximum discharge constraint that includes dependencies on both inflow and reservoir volume. Furthermore, we apply an algorithm (SDP) that explicitly handles non-convexities, separating our work from both [30] and [20]. We give a thorough description of the complicating elements of the constraint and elaborate on how to accurately model this type of state-dependent constraint in an SDP-based scheduling model.

The developed model is tested on a two-reservoir case study of a Norwegian hydro system. We discuss how the statedependent environmental constraint modify the resulting water value curves from the SDP algorithm, and use the water values to simulate operation of the system. The simulation results show the impact of the constraint on reservoir operation and economics. The novel contribution of this work is twofold in that we:

- Formulate a medium-term hydropower scheduling model based on SDP that accurately treats state-dependencies in the maximum allowed discharge from hydropower stations. Maximum discharge depends on the state variables reservoir volume, weekly inflow and a variable indicating if the low-inflow period has ended.

- Assess the impact of including such state-dependent maximum discharge constraints on the water value curves and shed light on the potential impacts that system operation guided by such curves may have. The assessment is carried out for a hydropower cascade in Norway.

The remainder of this paper is structured as follows: Section II describes the developed hydropower scheduling model; a case study is presented in Section III; and concluding remarks are found in Section IV. Section II comprises subsections describing the weekly decision problem (Section II-A), the statedependent environmental constraint on maximum discharge (Section II-B), the stochastic variables (Section II-C), and the solution strategy (Section II-D). The case study in III present calculated water values (Section III-B) and results from the simulations (Section III-C).

\section{Hydropower Scheduling Model}

In the following we present a medium-term hydropower scheduling model that is formulated for a hydropower cascade operated by a single hydropower producer assumed to be a risk-neutral price taker. The model maximises profit from operating the hydropower system for a presumed stationary future system state. The operation of the system is optimised for weekly decision stages over a horizon of one year.

The hydropower scheduling problem is a multi-stage stochastic optimisation problem. To solve the problem, we decompose the overall problem into several smaller subproblems, using the principles of SDP [11]. By decomposing the problem, we obtain one separate decision problem for each stage and state of the system. The SDP algorithm solves the decision problem, described in section II-A, for all stages and system states until convergence, as described in section II-D. The scope of potential system states is divided into a set of discrete states. The discrete states include all the information that is passed between the decision stages, from $t-1$ to $t$.
The set of states comprise subsets of endogenous states $\mathcal{S}^{p}$ and exogenous stochastic state variables $\mathcal{S}^{u}$. The storage volume in the reservoirs is the endogenous state variable. The stochastic state variables are: the weekly average energy price $\lambda$, the weekly total inflow into the system $\hat{Z}$ and the environmental state variable $\xi$. The stochastic variables are represented by a discrete Markov chain, as described in II-C. The environmental state variable indicates if the so called "low-inflow period" has ended. In practice the variable indicates whether the inflow level has exceeded a certain threshold over a shorter period of time. The extension of the discrete Markov chain to include an environmental state variable is explained further in section II-C, while the environmental constraint is described thoroughly in II-B. The implementation of the environmental constraint in the SDP algorithm is described in II-D.

\section{A. Decision Problem}

The decision problem is solved for a selection of given system states. Since the stochastic variables are known at the beginning of the stage, each single decision problem is solved as a deterministic problem. The stochastic nature of the problem is managed in the SDP algorithm. Uncertainty is represented through the price and inflow states, and the uncertainty of future realisations of the stochastic variables are reflected in the expected water values. Each stage is divided into $K$ number of time steps of constant length (hours).

The objective function (1) maximises the immediate profit of the decisions and the impact on the expected future profit given by $\alpha_{t+1}$. The expected future profit is a function of the stochastic state of the system and the resulting storage volume in the reservoirs at the end of the stage. Spillage of water is penalized according to a low cost $C^{S}$. Furthermore, operation of the reservoirs below a filling degree of $10 \%$ is penalized to represent risk-aversion of the producer.

$$
\begin{aligned}
& \alpha_{t}\left(s^{p}, s_{t}^{u}\right)= \\
& \quad \max \left\{F^{H} \lambda_{t} \sum_{k \in \mathcal{K}} \theta_{k} \sum_{h \in \mathcal{H}} p_{k, h}-C^{S} \sum_{k \in \mathcal{K}} \sum_{h \in \mathcal{H}} f_{k, h}\right. \\
& \left.-\frac{C^{R}}{F^{C}} \sum_{k \in \mathcal{K}} \sum_{h \in \mathcal{H}} \eta_{h, d=1} s_{k, h}^{r e s}+\alpha_{t+1}\left(v_{h \in H, k=K}, s_{t+1}^{u}\right)\right\}
\end{aligned}
$$

The energy production is a function of the discharge, $q_{k, h, d}$, from each of the reservoirs, as given in (2). The maximum and minimum discharge is limited by (3) and (4). Furthermore, the discharge is bounded by the availability of water in the reservoirs. The reservoir balance (5), keeps track of the change in water volume in each reservoir, where the volumes, $v_{k, h}$, are bounded by (6). To calibrate the reservoir management, a soft-constraint on minimum reservoir volume is used to reflect the risk-aversion of the producer.

$$
\begin{gathered}
p_{k, h}-\sum_{d \in \mathcal{D}_{h}} \eta_{h, d} q_{k, h, d}=0 \quad \forall k \in \mathcal{K}, h \in \mathcal{H} \\
q_{k, h, d} \leq Q_{h, d}^{\max } \quad \forall \quad k \in \mathcal{K}, h \in \mathcal{H}, d \in \mathcal{D}_{h}
\end{gathered}
$$




$$
\begin{gathered}
\sum_{d \in \mathcal{D}_{h}} q_{k, h, d} \geq Q_{h}^{\text {min }} \quad \forall k \in \mathcal{K}, h \in \mathcal{H} \\
v_{k, h}-v_{k-1, h}+F^{C}\left(\sum_{d \in \mathcal{D}_{h}} q_{k, h, d}+f_{k, h}\right) \\
-F^{C} \sum_{j \in \mathcal{H}_{h}^{u p}}\left(\sum_{d \in \mathcal{D}_{j}} q_{k, j, d}+f_{k, j}\right)=\phi_{k} Z_{h} \\
\quad \forall \quad k \in \mathcal{K} \backslash 1, h \in \mathcal{H} \\
V_{h}^{\text {min }}+0.1 *\left(V_{h}^{\text {max }}-V_{h}^{\text {min }}\right) \\
\leq v_{k, h}+s_{k, h}^{r e s} \leq V_{h}^{\text {max }} \quad \forall \quad k \in \mathcal{K}, h \in \mathcal{H}
\end{gathered}
$$

While (1)-(6) describes the general decision problem, the following equations present the interpolation in the expected future profit function for a cascaded system with two reservoirs. The expected future profit $\alpha_{t+1}$ is a function of the storage volume in the reservoirs at the end of the stage, and is bounded by (7)-(12). A two-dimensional, piecewise-linear approximation obtained by the triangle method is used to represent $\alpha_{t+1}$. The triangle method approximates functions of two variables by the use of linear triangles, see e.g. [32] and [33]. The method was chosen for its ability to approximate nonconvex functions and its simplicity [34].

The optimal expected future profit is obtained by convex combination of the expected future profit points $F V_{n, m}$ using the weighting variables $\gamma_{n, m}$, as given by (7)-(9). The expected future profit points are calculated for each of the discrete reservoir states in the previous stage, and given as input to the optimisation problem. The sum of the weighting variables in each dimension are used to find the total weight of the discrete volume segments for each reservoir in (10)-(11), binding the expected future profit to the storage volumes in the reservoirs at the end of the stage.

$$
\begin{gathered}
\alpha_{t+1}-\sum_{n \in \mathcal{N}} \sum_{m \in \mathcal{N}} \gamma_{n, m} F V_{n, m}=0 \\
\sum_{n=1}^{N} \sum_{m=1}^{N} \gamma_{n, m}=1 \\
\gamma_{n, m} \leq 1, \quad \forall \quad n \in \mathcal{N}, m \in \mathcal{N} \\
\beta_{h, n}=\sum_{m \in \mathcal{N}} \gamma_{n, m} \quad \forall \quad h \in \mathcal{H}, n \in \mathcal{N} \\
v_{k, h}-\sum_{n \in \mathcal{N}} \beta_{h, n} V_{h, n}^{\text {seg }}=0 \quad \forall \quad k=K, h \in \mathcal{H} \\
\chi_{g}=\sum_{\{n, m\} \in \mathcal{N}_{g}^{\text {Diag }}} \gamma_{n, m} \quad \forall \quad g \in \mathcal{G} \\
p, v, q, f, \gamma, s^{\text {res }} \in \mathbb{R}^{+}, \quad \alpha_{t+1} \in \mathbb{R}
\end{gathered}
$$

Nonconvex characteristics in the expected future profit function are dealt with by restricting the weighing variables $(\gamma)$.
In the optimal solution, maximum two adjacent weights $(\gamma)$ in each dimension can have non-zero values, thereby forming a square of adjacent weighting variables that can be active. Such behaviour can be enforced by special ordered sets of type 2 (SOS2) [35]. In SOS2, only two adjacent variables in the set can be non-zero. SOS2 are included in most commercial solvers, such as CPLEX, which is used in this work. In (14), $\beta_{h}$ is defined as one $\operatorname{SOS} 2$ for each dimension $h$ (i.e. for each reservoir). By using four weights to describe a point in two dimensions, the model is given the freedom to decide which points to use. To ensure one unique solution, we force the model to use a predefined set of weights (3 out of 4) by defining the set $\chi$ to be a SOS2 in (15) [35]. The set $\chi$ comprise the sum of the weights $\gamma$ in the diagonal direction, hence forming a triangle of adjacent weighting variables. The SOS2 defined in (14) and (15) can be removed if the future profit function is concave, changing the formulation from an MILP to an LP.

$$
\beta_{h} \text { SOS-2 } \forall h \in \mathcal{H}
$$

$\chi$ SOS-2

\section{B. Activation of the Environmental Regulation}

Time and state-dependent restrictions on maximum discharge from the reservoirs are one special type of environmental constraint which is found in the regulation of several Norwegian hydropower plants. The activation and/or deactivation criteria of these constraints are often formulated as logical conditions dependent on the state variables, i.e. storage volume in the reservoirs and inflow. Time-dependent maximum discharge capacities can be implemented in the hydropower scheduling model without difficulties, while formulations that include state-dependencies can present modelling challenges as discussed in section I. Here, we present a general formulation of the regulation. The dependencies on both inflow and storage volume in the reservoir are handled in the SDP algorithm, as described in II-D. The maximum discharge regulation is given as follows:

- Other than to honour minimum flow obligations, no discharge is allowed within a given period of time, unless the storage volume in the reservoir reach a given threshold. If the required threshold is reached, discharge is permitted as long as the storage volume is kept above the threshold.

- The constraint can be activated earlier by inflow levels above a given threshold over a short period.

- From a certain date and until the end of the restriction period, discharge from the reservoir is permitted as long as the storage volume in the reservoir does not decrease.

The above regulation can be expressed mathematically by a set of constraints. The different constraints are activated by predefined logical conditions. The state-dependent conditions are illustrated in Fig. 1.

The different type of constraints imposed by the maximum discharge regulation are described by (16)- (19). Depending on the current state of the system, the constraints are added to 


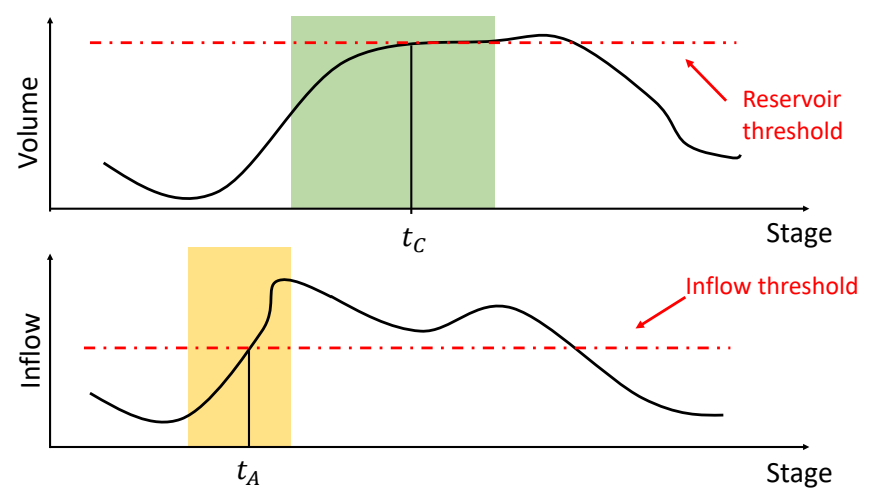

Fig. 1. Illustration of the state-dependent conditions in the environmental regulation. Within the orange shaded period, the constraint can be activated by inflow above a defined threshold, illustrated by point $t_{A}$. Similarly, the no discharge restriction can be deactivated within the green shaded area by storage volume above the reservoir threshold, as illustrated by point $t_{C}$.

the formulation before the decision problem is solved. The first part of the environmental regulation is a maximum discharge capacity constraint. The constraint is shown in (16), where only a minimum discharge flow is allowed. The constraint is activated by inflow above a certain threshold (in $t_{A}$ ) or by a given date, $t_{B}$.

$$
\sum_{d \in \mathcal{D}_{h}} q_{k, h, d} \leq Q_{h}^{\min } \quad \forall k \in \mathcal{K}, h=\hat{H}
$$

When (and if) the storage volume in the reservoir reach the predefined threshold, the constraint changes into a minimum reservoir level constraint. This can be imposed by (17) and (18). (17) is active for the first week the storage volume in the reservoir reach the wanted threshold (in $t_{C}$ ), while (18) becomes active from the following week.

$$
\begin{gathered}
v_{k=K, h} \geq V_{h}^{l i m} \quad \forall h=\hat{H} \\
v_{k, h} \geq V_{h}^{l i m} \quad \forall \quad k \in \mathcal{K}, h=\hat{H}
\end{gathered}
$$

Finally, for a given period, $t_{D}-t_{E}$, the storage volume in the reservoir is not permitted to decrease. This constraint is shown in (19). The formulation ensures that the storage level in the reservoir at the end of a stage is equal or higher than the storage level at the beginning of the stage. The storage level could also be bounded over each time step $k$. While we did not encounter feasibility issues, such can be avoided using a slack-variable that is penalised in the objective function.

$$
v_{k, h} \geq V_{h} \quad \forall \quad k=K, h=\hat{H}
$$

\section{Stochastic variables}

We consider three exogenous stochastic variables in this work; the total weekly inflow into the system, the weekly average energy price and the environmental state, i.e. if the inflow has been above a certain threshold. Inflow normally has a strong weekly correlation, while inflow and price tend to be negatively correlated in hydro-dominated systems. The environmental state variable is an extension of the inflow state. For computational simplicity, we assume that the stochastic variables can be described by discrete nodes using a Markov decision process. The following procedure is used to generate the Markov chain:

- Inflow and price data is given as input to the model, e.g. historical data.

- An auto-regressive model is fitted to the input data. Serial correlation in inflow and cross-correlation between inflow and price is considered.

- 10000 scenarios are sampled from the auto-regressive model.

- A given number of discrete nodes per week are generated from the scenarios, using K-means clustering. Each node represents one inflow value and one price value.

- The transition probabilities are determined by counting the share of scenarios transitioning between the different nodes from one week to the next.

In addition, information on the environmental state is required. The environmental state represents a binary variable, indicating whether the inflow has been above a certain threshold. For the weeks when this is applicable, the Markov chain is expanded with an additional environmental state (activated and not activated) in each of the nodes, as illustrated in Fig. 2. The transition probabilities are updated by multiplying with the probability of inflow above (or below) the threshold in each node, presuming that the inflow level has not previously been above the threshold. Once the environmental state is activated, all future environmental states has to be active as well.

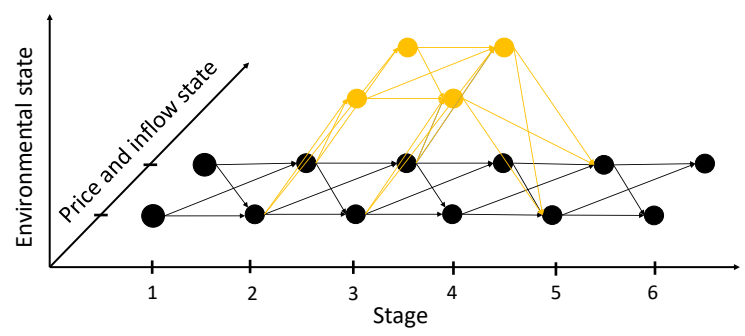

Fig. 2. Illustration of a Markov chain with two nodes per stage representing unique price and inflow values, and an additional environmental state in $t=3$ to 4 . The additional nodes are illustrated in yellow.

\section{Solution Strategy}

The hydropower scheduling problem is solved using the SDP algorithm described in Algorithm 1. The algorithm is based on backwards recursion and solves the decision problem for each stage and state of the system until convergence. The algorithm iterates over all stages $(\mathcal{T})$, all reservoir states $\left(\mathcal{S}^{p}\right)$, and all stochastic states $\left(\mathcal{S}^{u}\right)$ in lines 4-6. $\mathcal{S}^{p}$ comprises all combinations of discrete storage volumes for the reservoirs in the system. The stochastic variables are updated in line 7, while reservoir specific data is updated for each hydropower plant in lines 8-11. The expected future profits for all end reservoir states are updated in line 12 . 


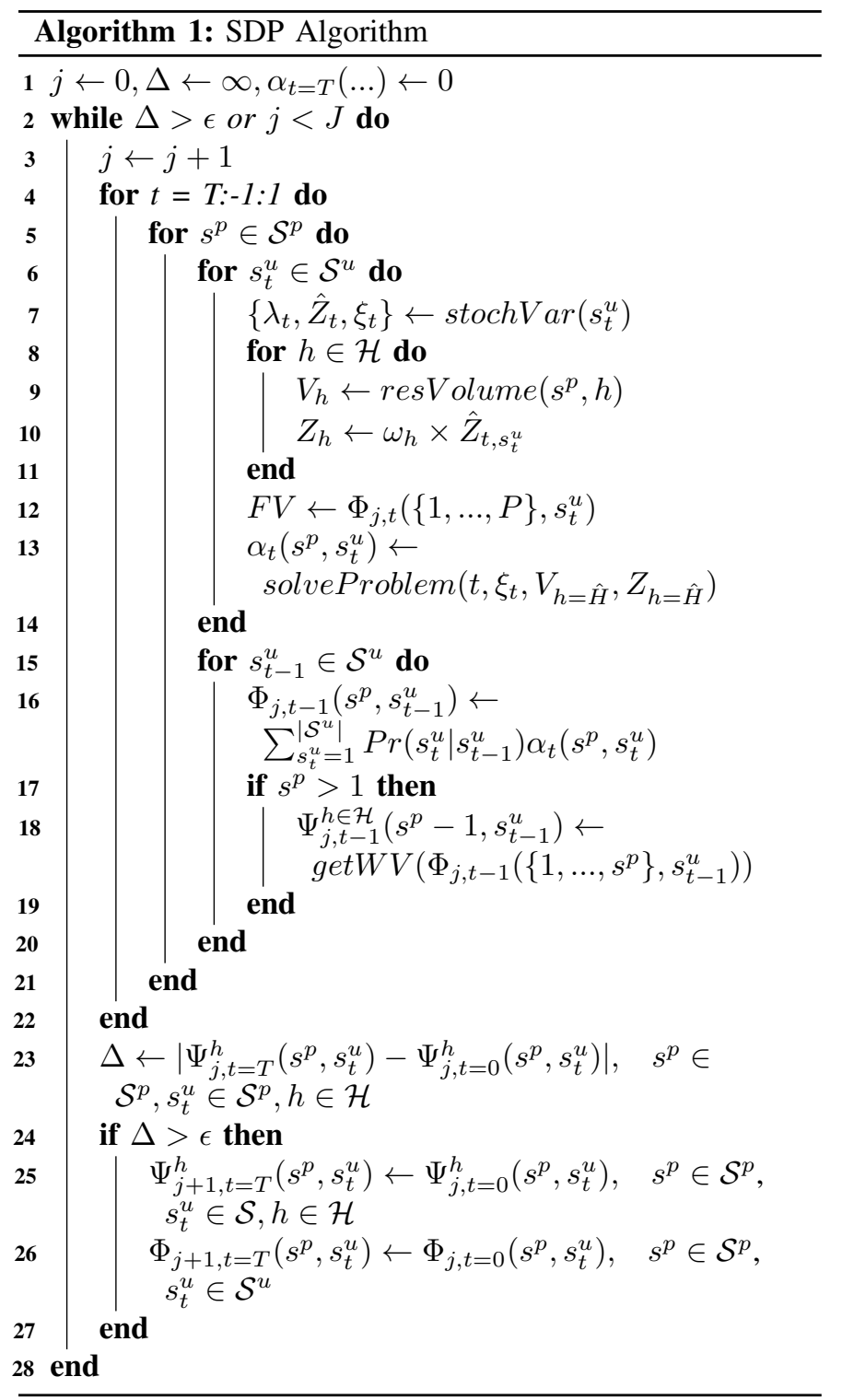

The decision problem is solved in line 13 following the procedure described in algorithm 2 . The algorithm checks if any of the environmental conditions described in section II-B are met, and solves the associated decision problem with the corresponding constraints included, i.e (16), (17), (18) or (19). The inflow dependent early activation of the constraint is included as a stochastic state $\xi$ in the discrete Markov chain. If the problem is solved for a system without the environmental regulation, the decision problem is described by (1)-(13). Without the environmental constraints, the expected future profit function is concave, and (14) and (15) can be relaxed.

The solution of the optimisation problem for all stochastic states $s_{t}^{u}$ is used to calculate the expected future profit, in line 16. The expected future profit is stored to matrix $\Phi$. The water values are calculated and stored to the water value matrix $\Psi$ in line 18, following a similar approach as in [18]. Convergence is determined in line 23 , by comparing the calculated water values in the last and first stage. If the algorithm has not

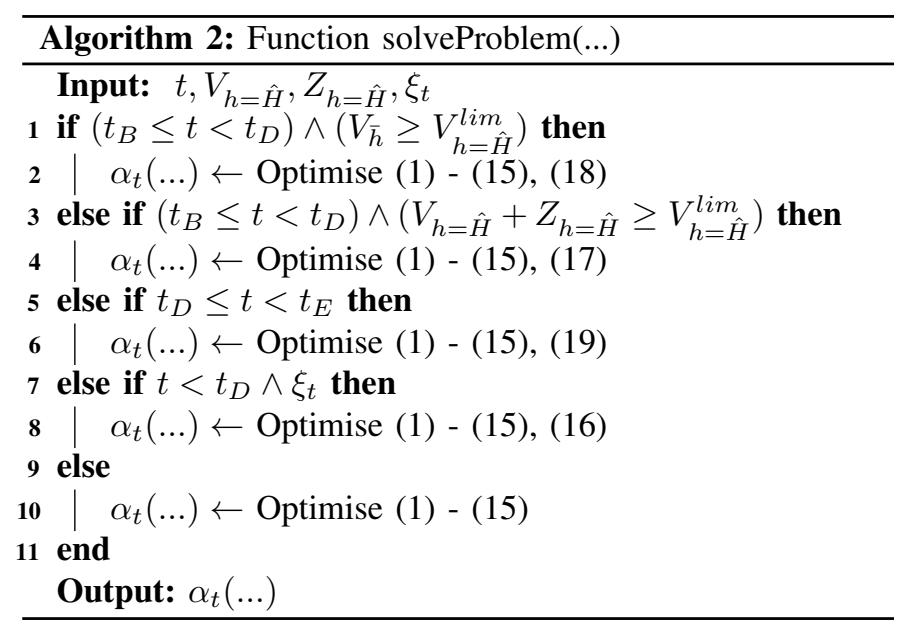

converged in iteration $j$, the water value matrix and the expected future profit matrix for the last stage $T$ is updated in lines 25 and 26 before the next iteration $j+1$ starts.

\section{CASE STUdY}

\section{A. Case Description}

This case study assesses the impact of the state-dependent maximum discharge regulation on water values, simulated reservoir operation and profit from the simulated operation of the system. First, water values are calculated in the SDP model, before optimal operation of the system is simulated for a selection of scenarios.

The described model is applied to the hydropower system shown in Fig. 3. The hydropower system is based on the Bergsdalen watercourse in Western Norway. The system comprise several hydropower reservoirs and power plants, of which the two upper reservoirs and power plants are modelled here. The modelled part of the system has a total generation capacity of approximately $55 \mathrm{MW}$ and a reservoir storage capacity of up to $360 \mathrm{Mm}^{3}$.

The lower of the two modelled reservoirs has a statedependent environmental maximum discharge constraint. The constraint is of the type described in II-B. The discharge limitation can be activated by inflow above the weekly average from week 15 and at the latest in week 19. The discharge limitation is active until week 32 , or until the storage volume in the reservoir is above $146 \mathrm{Mm}^{3}$. If the storage volume in the reservoir reach $146 \mathrm{Mm}^{3}$, discharge is permitted as long as the water level stays above the threshold. From week 33 to week 35 the reservoir storage is not allowed to be reduced. The constraint is deactivated in week 35 . In addition, a minimum discharge of $3 \mathrm{~m}^{3} / \mathrm{s}$ is imposed on the lower reservoir.

Two cases are considered; without the environmental regulation, wo/Env, and with the environmental regulation, $w / E n v$. In the $w / E n v$ case, the decision problem is solved with the environmental regulation, as given in section II-D. Due to the nonconvex characteristics of the environmental constraint, (14) and (15) are required, making the problem an MILP. In the $w o / E n v$ case, the problem is an LP described by (1) - (13).

The SDP model is solved for one year of weekly decision stages, comprising three price periods of 56 hours each. 


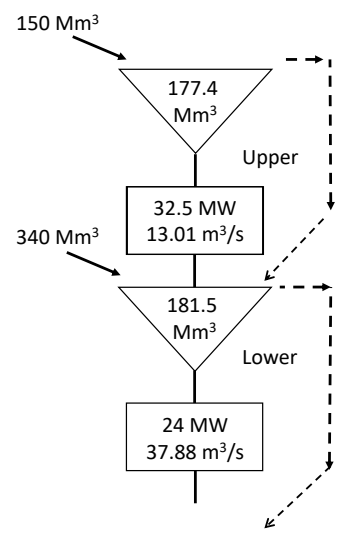

Fig. 3. Topology of the modelled system. Reservoirs (triangles), power plants (rectangles), and water routes for discharge (solid lines) and spillage (dashed lines) are shown. Maximal values for discharge $\left(\mathrm{m}^{3} / \mathrm{s}\right)$, production (MW), reservoir volumes $\left(\mathrm{Mm}^{3}\right)$ and average yearly inflow $\left(\mathrm{Mm}^{3}\right)$ are given (not considering the environmental constraint).

A high intra-week price variation is assumed. Each of the reservoirs are discretised into 20 equidistant points, giving 400 combinations of reservoir states. Since the focus of this work is on the modelling of the environmental constraint, a relatively coarse representation of uncertainty and time discretisation is included. A discrete Markov chain with 10 nodes per stage is used, each comprising a unique price and inflow value. In the weeks when early activation of the environmental constraint could occur, the environmental state variable is added, leading to a total of 20 nodes per stage. The discrete Markov chain is generated using inflow data from 58 historical years and power prices generated based on the same inflow data. The power prices were provided from the long-term hydropower scheduling model EMPS [25], based on a low emission dataset of the European power system for year 2030 [36]. The penalty cost of spilling water is set low, $C^{S}=10^{-3} \frac{€}{\mathrm{~m}^{3} / \mathrm{s}}$, while the cost of drawing down the reservoir below $10 \%$ of the storage capacity is set slightly higher than two times the average power price, $C^{R}=78 \frac{€}{M W h}$.

The simulation is conducted as weekly decisions in a sequence, solving the decision problem formulated in II-A as a short-term operational problem for each week. The same technical details as in the SDP model is included. The water values calculated in the SDP model are used as input to the weekly decision problem to evaluate the value of the water in the reservoir at the end of each week. For the $w / E n v$ case, the logical conditions for activating the environmental constraint, as described in II-B, are checked for each time the weekly decision problem is solved. When (and if) the conditions are met, the associated constraints, i.e. (16), (17), (18) or (19), are added to the decision problem.

The model was implemented in Julia v1.5 using the Jump package [37] and the CPLEX 12.10 solver [38]. The relative MIP gap is set to zero and the absolute MIP gap to $10^{-10}$. The topic of computation time is not considered, as the SDP methodology mainly was chosen to enable a nonconvex model. The convergence criterion in the SDP algorithm is set to $10^{-3} \frac{€}{M m^{3}}$.

\section{B. Water Values}

The water values are the main result from the SDP model. In our work, we are especially interested in how the water values change when including the state-dependent maximum discharge constraint. This is of high importance in real life hydropower scheduling, as the water values provide essential information into short-term decision making.

The results and discussion of this section only consider the water values of the lower reservoir, since small or no changes were seen in the water values of the upper reservoir in this case study. We assume a medium storage volume in the upper reservoir (i.e. $85 \mathrm{Mm}^{3}$ ).

Fig. 4 shows the calculated water values for the wo/Env case and the $w / E n v$ case. The water values change with the storage volume in the reservoir and the week of the year. In the $w o / E n v$ case, the water values are non-increasing with increasing storage volume in the reservoir. This will always be the result from linear hydropower scheduling models where the expected future profit is a concave function. For low storage volumes in the reservoir, the water value is high because of the risk of emptying the reservoir. For higher storage volumes in the reservoir, the water value decrease as the risk of spilling water increase. The water value is zero when water has to be spilled because of full reservoirs. In the $w / E n v$ case, the same behaviour of high and low water values can be observed for low and high storage volumes respectively. However, the water values also sometimes increase with increasing storage volumes. For this case, the expected future profit is therefore a nonconcave function.

The increasing water values with increasing reservoir storage volumes in the $w / E n v$ case are a direct result of the state-dependent maximum discharge constraint. The largest differences in the water values between the two cases can be seen when the strictest part of the environmental regulation is active (week 18 to 32) and backwards in time (towards week 1). In the weeks when the constraint is active and the storage volume in the reservoir is lower than the threshold $\left(146 \mathrm{Mm}^{3}\right)$, the water values are higher in the $w / E n v$ case than in the wo/Env case. Since discharge from the reservoir is strictly limited over a longer period, the model cannot take advantage of the potential high prices within this period, unless the threshold is reached. As a result, the water values are higher in the $w / E n v$ case compared to the wo/Env case for storage volumes where the maximum discharge capacity restriction can be deactivated early.

The water values are calculated from the last to the first week of the year, hence the impact of the constraint on the water values from later weeks affects the earlier weeks. Week 32 is the last week when the maximum discharge capacity restriction is active. Fig. 5 compares the water values from the two cases for selected weeks. No large differences can be seen after the constraint is deactivated, this is for example shown in Fig. 5a. In week 32 (Fig. 5b), the difference between the 


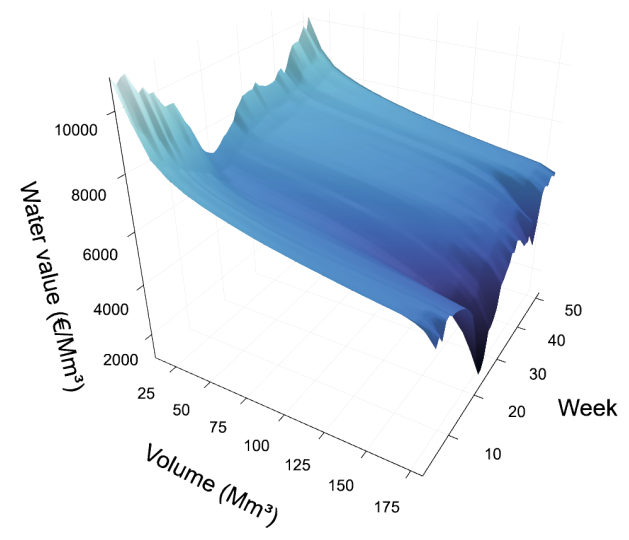

(a) wo/Env

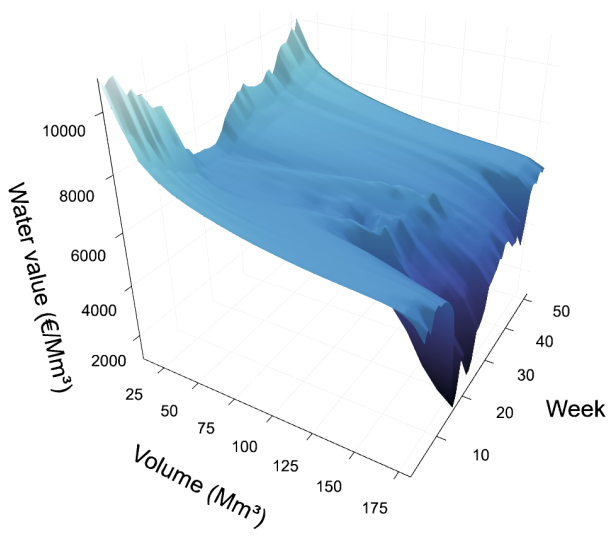

(b) w/Env

Fig. 4. Calculated water values for the lower reservoir in the two cases plotted for the storage volume in the reservoir and week of the year.

water value curves for the two cases becomes more distinct. The local peak in the water value curve for the $w / E n v$ case in Fig. $5 \mathrm{~b}$ is a result of discharge being permitted when the storage volume in the reservoir reach the threshold. Moving forward in time, this effect is being shifted towards lower storage volumes, as shown in Fig. 5c and Fig. 5d.

\section{Simulation Results}

Simulations were run for 1000 scenarios, randomly selected from the originally sampled scenarios. The wo/Env case and the $w / E n v$ case were simulated for the same scenarios, using water values from the SDP model for each of the cases accordingly. The final simulations are conducted to test the calculated water values in an operational decision making setting, as well as to demonstrate the effect of the environmental constraint on operation of the system.

The activation and deactivation of the maximum discharge capacity restriction varies between the scenarios because of the state-dependent nature of the conditions. Fig. 6 shows when the maximum discharge constraint is activated and deactivated in the $w / E n v$ case for the simulated scenarios. In approximately $50 \%$ of the scenarios, the discharge limitation is activated early by high inflow, while the remaining $50 \%$ is forcibly activated in week 19. Similarly, the storage volume in approximately $85 \%$ of the scenarios reach the reservoir threshold before week 33, deactivating the maximum discharge constraint.

The results from the simulations show a considerable change in optimal operation when the environmental constraint is included. Fig. 7 compare the change in storage volume in the lower reservoir over a year for the $w o / E n v$ and $w / E n v$ case. In general, the storage volume in the reservoir is kept higher in the $w / E n v$ case. The largest difference can be seen in the spring and summer weeks, when the environmental regulation is active. For several weeks in this period, the median reservoir storage volume is raised from below $100 \mathrm{Mm}^{3}$ in the wo/Env case to over $150 \mathrm{Mm}^{3}$ in the $w / E n v$ case, demonstrating the effectiveness of the constraint.
For most of the simulated scenarios, the storage volume in the lower reservoir reach the reservoir threshold in week 25 to 30 in the $w / E n v$ case. This means that the optimal operation of the reservoirs lies within the reservoir volume segments where the maximum discharge capacity constraint has the largest impact on the water values, indicating the importance of including the constraint in the calculation of the water values. Furthermore, the reservoir storage volume is also kept higher in the autumn and winter weeks in the $w / E n v$ case compared to the $w o / E n v$ case. Higher storage volumes throughout the year, and not only when the constraint on discharge is active, can be explained by the differences in water values. Higher water values for higher storage volumes in the $w / E n v$ case give the model an incentive to keep more water in the reservoir when coming into the constraint period.

A selection of average numeric results from the completed simulations are given in Table III-C. The total yearly profit from the system is reduced by around $72 \mathrm{k} €(0.8 \%)$ in the $w / E n v$ case compared to the $w o / E n v$ case. Over $90 \%$ of this loss comes from the lower reservoir. In the period when the constraint is active, week 19-35, the total profit from electricity generation is reduced by approximately $17 \%$ or 406 $\mathrm{k} €$. By shifting the electricity generation, the model manages to keep the total generation in the $w / E n v$ case close to the total generation in the $w o / E n v$ case, significantly reducing the total loss in profit. This means that the loss in profit mainly is caused by a lower average realised price of electricity, and not reduced sales of electricity. Still, it should be mentioned that the economic results are sensitive to the power price assumptions used in the simulations. Higher power prices in the constrained period can increase the cost of restricting production in this period, and vice versa.

\section{CONCLUSION}

In this work, we have developed a medium-term hydropower scheduling model comprising a state-dependent environmental constraint on maximum discharge, and applied the model to a case study of a Norwegian hydropower system with multiple 


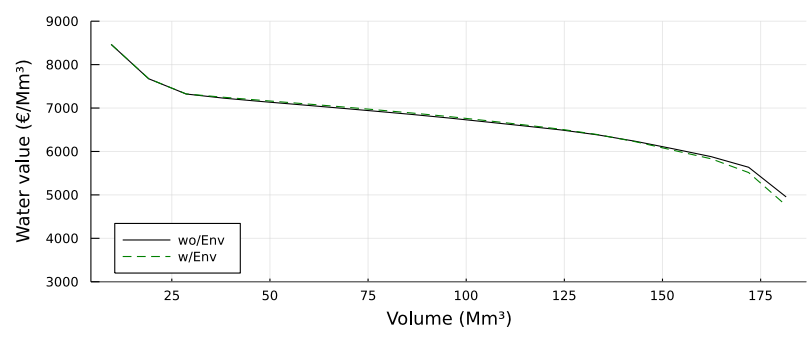

(a) Week 33

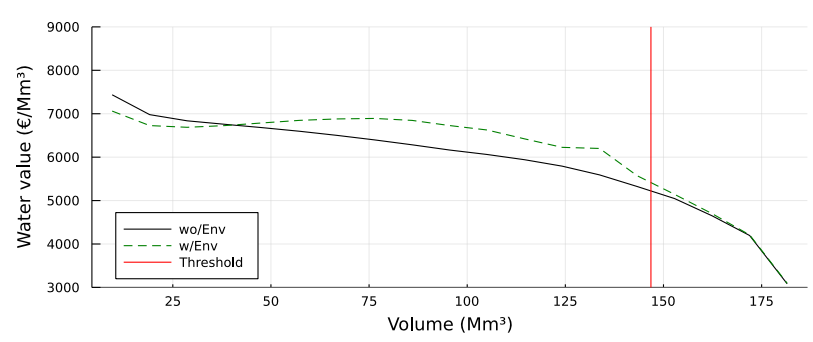

(c) Week 25

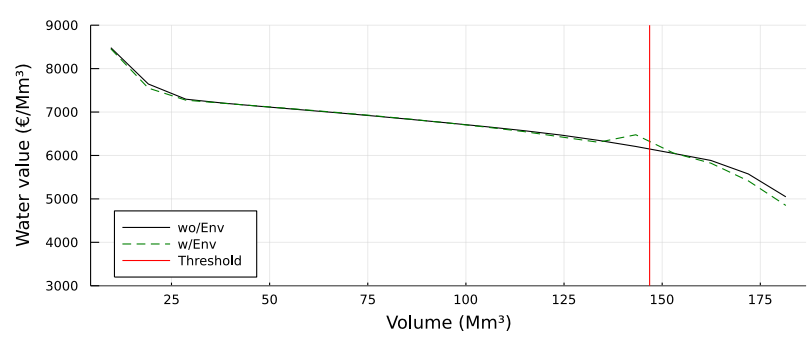

(b) Week 32

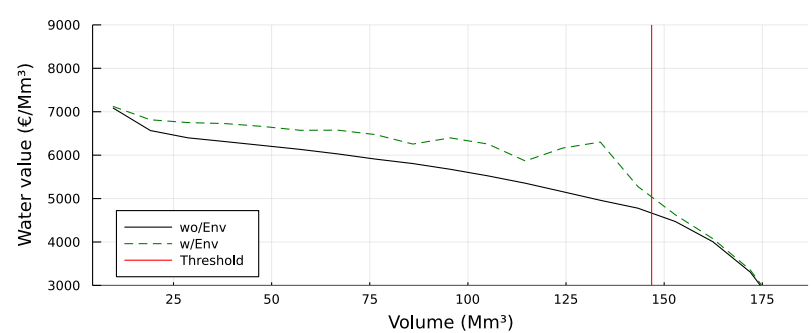

(d) Week 20

Fig. 5. Water values calculated in the $w / E n v$ case (dashed lines) and the $w o / E n v$ case (solid lines). The vertical lines give the reservoir threshold.

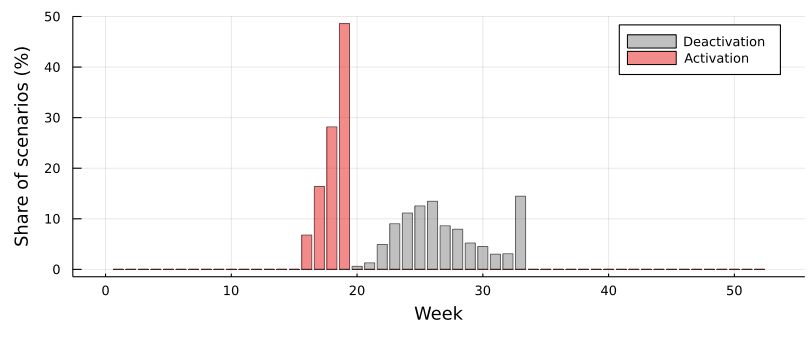

Fig. 6. Activation and deactivation of the maximum discharge restriction per week, given in share of scenarios.

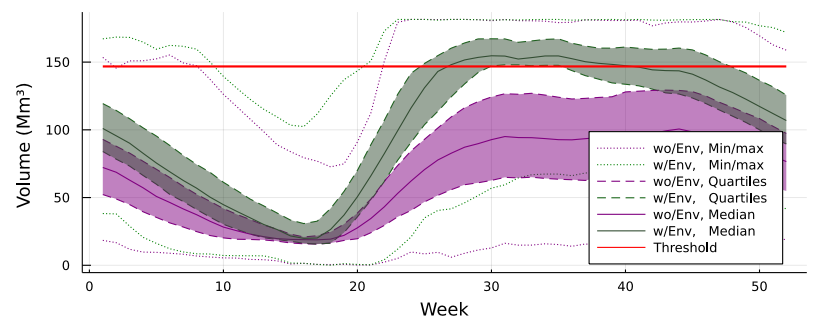

Fig. 7. Simulated storage volume in the lower reservoir in the wo/Env (purple) and $w / E n v$ (green) cases. Min/max (dotted lines), quartiles (dashed lines), and the medians (solid lines) are shown. The horizontal line gives the reservoir threshold.

reservoirs. Simulations of the system with and without the environmental constraint show a substantial difference in operation of the reservoir to which the constraint is imposed. In the case with the environmental constraint, the storage volume in the reservoir reach the wanted threshold for most of the scenarios. Still, the total electricity generation over the year is maintained and total loss in profit limited to approximately $0.8 \%$.

The calculated water values were found to change considerably when the state-dependent maximum discharge constraint
TABLE I

AVERAGE PROFIT AND ELECTRICITY GENERATION

\begin{tabular}{|ll|lll|}
\hline Case & Reservoir & Profit $[€ / \mathrm{yr}]$ & $\begin{array}{l}\text { Production } \\
{[\mathrm{MWh} / \mathrm{yr}]}\end{array}$ & $\begin{array}{l}\text { Profit week } \\
19-35[€]\end{array}$ \\
\hline \multirow{3}{*}{ wo/Env } & Upper & $5.16 \mathrm{E}+06$ & $1.04 \mathrm{E}+05$ & $1.20 \mathrm{E}+06$ \\
& Lower & $4.19 \mathrm{E}+06$ & $9.00 \mathrm{E}+04$ & $1.16 \mathrm{E}+06$ \\
& Total & $9.34 \mathrm{E}+06$ & $1.94 \mathrm{E}+05$ & $2.36 \mathrm{E}+06$ \\
\hline \multirow{3}{*}{ w/Env } & Upper & $5.15 \mathrm{E}+06$ & $1.04 \mathrm{E}+05$ & $1.24 \mathrm{E}+06$ \\
& Lower & $4.12 \mathrm{E}+06$ & $8.98 \mathrm{E}+04$ & $7.16 \mathrm{E}+05$ \\
& Total & $9.27 \mathrm{E}+06$ & $1.94 \mathrm{E}+05$ & $1.95 \mathrm{E}+06$ \\
\hline \multirow{2}{*}{ Difference } & Upper & $-0.10 \%$ & $-0.0 \%$ & $3.12 \%$ \\
& Lower & $-1.60 \%$ & $-0.22 \%$ & $-38.46 \%$ \\
& Total & $-0.77 \%$ & $-0.11 \%$ & $-17.23 \%$ \\
\hline
\end{tabular}

was included in the SDP model. For the reservoir to which the constraint was imposed, the water values were found to both increase and decrease with increasing storage volumes in the reservoir, reflecting that the expected future profit function is nonconcave. The distinct changes in the calculated water values when the environmental regulation was considered show the importance of accurate modelling of such regulations. This conclusion is further strengthened by the optimal reservoir operation being within the nonconcave area of the expected future profit function for most of the simulated scenarios, as demonstrated by the simulation results. If the future profit function is used as boundary condition in operational shortterm scheduling models, the nonconcave shape may impact the results substantially.

To the best of our knowledge, most used medium-term hydropower scheduling models require a convex model formulation, and can therefore not be used without further enhancements to obtain accurate water values considering the impact of logical conditions and state-dependent constraints. The substantial impact on the calculated water values and reservoir operation observed in this study, support further research on nonconvex environmental constraints. To strengthen the 
findings from this study, the impact of using accurate water values, compared to water values based on simplified problem formulations, in operation of hydropower systems with environmental regulation should be investigated. Furthermore, a wider selection of cases and hydropower watercourses with different variations of the state-dependent maximum discharge constraint, could be analysed. Finally, a broader analysis to evaluate how well the constraint meets the underlaying purpose of the environmental regulation, as well as other unintended consequences like flood risk could be of interest.

\section{ACKNOWLEDGMENT}

The authors would like to thank our colleagues Kasper Thorvaldsen, Emil Dimanchev and Gro Klæboe for their contributions to the original manuscript.

\section{REFERENCES}

[1] "The European Green Deal," https://ec.europa.eu/info/strategy/ priorities-2019-2024/european-green-deal_en, last accessed 03.06.2021.

[2] M. Mattmann, I. Logar, and R. Brouwer, "Hydropower externalities: A meta-analysis," Energy Economics, vol. 57, pp. 66-77, 2016.

[3] N. L. Poff and J. K. Zimmerman, "Ecological responses to altered flow regimes: A literature review to inform the science and management of environmental flows," Freshwater Biology, vol. 55, no. 1, pp. 194-205, 2010.

[4] B. Köhler, A. Ruud, Ø. Aas, and D. N. Barton, "Decision making for sustainable natural resource management under political constraints the case of revising hydropower licenses in Norwegian watercourses," Civil Engineering and Environmental Systems, vol. 36, no. 1, pp. 17-31, 2019.

[5] L.-R. D. Kosnik, "Balancing Environmental Protection and Energy Production in the Federal Hydropower Licensing Process," SSRN Electronic Journal, 2011.

[6] I. Guisández, J. I. Pérez-Díaz, and J. R. Wilhelmi, "Assessment of the economic impact of environmental constraints on annual hydropower plant operation," Energy Policy, pp. 1332-1343, 2013.

[7] E. G. Dimanchev, J. L. Hodge, and J. E. Parsons, "The role of hydropower reservoirs in deep decarbonization policy," Energy Policy, vol. 155 , pp.,- 82021.

[8] A. Helseth and C. G. de Melo, "Scheduling Toolchains in HydroDominated Systems - Evolution, Current Status and Future Challenges," SINTEF, Trondheim, Tech. Rep., 2020

[9] M. V. Pereira and L. M. Pinto, "Multi-stage stochastic optimization applied to energy planning," Mathematical Programming, vol. 52, no. 1-3, pp. 359-375, 1991.

[10] A. Gjelsvik, M. Belsnes, and A. Haugstad, "An algorithm for stochastic medium-term hydrothermal scheduling under spot price uncertainty." Proceedings. 13th Power Systems Computation Conference, 1999, Vol.2, pp. 1079-1085, 1999.

[11] R. Bellman, "Dynamic programming and stochastic control processes," Information and Control, vol. 1, no. 3, pp. 228-239, 91958.

[12] A. L. Diniz and M. E. Piñeiro Maceira, "A four-dimensional model of hydro generation for the short-term hydrothermal dispatch problem considering head and spillage effects," IEEE Transactions on Power Systems, vol. 23, no. 3, pp. 1298-1308, 2008.

[13] W. van Ackooij, I. Danti Lopez, A. Frangioni, F. Lacalandra, and M. Tahanan, "Large-scale unit commitment under uncertainty: an updated literature survey," Annals of Operations Research, vol. 271, no. 1, pp. 11-85, 122018

[14] A. Helseth, M. Fodstad, and B. Mo, "Optimal Medium-Term Hydropower Scheduling Considering Energy and Reserve Capacity Markets," IEEE Transactions on Sustainable Energy, vol. 7, no. 3, pp. 934 942, 72016

[15] S. Cerisola, J. M. Latorre, and A. Ramos, "Stochastic dual dynamic programming applied to nonconvex hydrothermal models," European Journal of Operational Research, vol. 218, no. 3, pp. 687-697, 52012.

[16] Q. Goor, R. Kelman, and A. Tilmant, "Optimal MultipurposeMultireservoir Operation Model with Variable Productivity of Hydropower Plants," Journal of Water Resources Planning and Management, vol. 137, no. 3, pp. 258-267, 52011.
[17] E. Pereira-Bonvallet, S. Püschel-Løvengreen, M. Matus, and R. Moreno, "Optimizing Hydrothermal Scheduling with Non-Convex Irrigation Constraints: Case on the Chilean Electricity System," in Energy Procedia, vol. 87. Elsevier Ltd, 2016, pp. 132-140.

[18] A. Helseth, M. Fodstad, M. Askeland, B. Mo, O. B. Nilsen, J. I. PérezDíaz, M. Chazarra, and I. Guisández, "Assessing hydropower operational profitability considering energy and reserve markets," IET Renewable Power Generation, vol. 11, no. 13, pp. 1640-1647, 112017.

[19] M. N. Hjelmeland, J. Zou, A. Helseth, and S. Ahmed, "Nonconvex Medium-Term Hydropower Scheduling by Stochastic Dual Dynamic Integer Programming," IEEE Transactions on Sustainable Energy, vol. 10, no. 1, pp. 481-490, 2019.

[20] A. Helseth, B. Mo, and H. O. Hågenvik, "Nonconvex Environmental Constraints in Hydropower Scheduling," in International Conference on Probabilistic Methods Applied to Power Systems (PMAPS), 2020.

[21] J. D. C. Little, "The Use of Storage Water in a Hydroelectric System," Journal of the Operations Research Society of America, vol. 3, no. 2, pp. 187-197, 51955.

[22] J. Lindqvist, "Operation of a Hydrothermal Electric System: A Multistage Decision Process," Transactions of the American Institute of Electrical Engineers. Part III: Power Apparatus and Systems, vol. 81, no. 3, pp. 1-6, 1962.

[23] J. A. Tejada-Guibert, S. A. Johnson, and J. R. Stedinger, "Comparison of two approaches for implementing multireservoir operating policies derived using stochastic dynamic programming," Water Resources Research, vol. 29, no. 12, pp. 3969-3980, 121993.

[24] A. Turgeon and R. Charbonneau, "An aggregation-disaggregation approach to long-term reservoir management," Water Resources Research, vol. 34, no. 12, pp. 3585-3594, 121998.

[25] O. Wolfgang, A. Haugstad, B. Mo, A. Gjelsvik, I. Wangensteen, and G. Doorman, "Hydro reservoir handling in Norway before and after deregulation," Energy, vol. 34, no. 10, pp. 1642-1651, 102009.

[26] M. A. Olivares, J. Haas, R. Palma-Behnke, and C. Benavides, "A framework to identify Pareto-efficient subdaily environmental flow constraints on hydropower reservoirs using a grid-wide power dispatch model," Water Resources Research, vol. 51, no. 5, pp. 3664-3680, 2015.

[27] I. Guisández, J. I. Pérez-Díaz, and J. R. Wilhelmi, "Approximate formulae for the assessment of the long-term economic impact of environmental constraints on hydropeaking," Energy, vol. 112, pp. 629641, 2016.

[28] S. Steinschneider, A. Bernstein, R. Palmer, and A. Polebitski, "Reservoir Management Optimization for Basin-Wide Ecological Restoration in the Connecticut River," Journal of Water Resources Planning and Management, vol. 140, no. 9, p. 04014023, 92014.

[29] L. E. Schäffer, A. Adeva-Bustos, T. H. Bakken, A. Helseth, and M. Korpas, "Modelling of Environmental Constraints for Hydropower Optimization Problems - a Review," in 2020 17th International Conference on the European Energy Market (EEM). IEEE, 9 2020, pp. $1-7$.

[30] I. Guisández, J. I. Pérez-Díaz, W. Nowak, and J. Haas, "Should environmental constraints be considered in linear programming based water value calculators?" International Journal of Electrical Power and Energy Systems, vol. 117, 2020.

[31] I. Guisández, J. I. Pérez-Díaz, and J. R. Wilhelmi, "The influence of environmental constraints on the water value," Energies, vol. 9, no. 6, 2016.

[32] I. Guisández and J. I. Pérez-Díaz, "Mixed integer linear programming formulations for the hydro production function in a unit-based shortterm scheduling problem," International Journal of Electrical Power and Energy Systems, vol. 128, p. 106747, 62021.

[33] R. Misener and C. A. Floudas, "Piecewise-Linear Approximations of Multidimensional Functions," J Optim Theory Appl, vol. 145, pp. 120$147,2010$.

[34] C. D'Ambrosio, A. Lodi, and S. Martello, "Piecewise linear approximation of functions of two variables in MILP models," Operations Research Letters, vol. 38, no. 1, pp. 39-46, 12010.

[35] P. H. Williams, Model Building in Mathematical Programming, 5th Edition - Wiley. West Sussex: Wiley, 2013, vol. 5th Edition.

[36] L. E. Schäffer, B. Mo, and I. Graabak, "Electricity Prices and Value of Flexible Generation in Northern Europe in 2030," in International Conference on the European Energy Market, EEM, 2019.

[37] I. Dunning, J. Huchette, and M. Lubin, "JuMP: A Modeling Language for Mathematical Optimization," SIAM Review, vol. 59, no. 2, pp. $295-$ $320,82015$.

[38] "IBM CPLEX optimizer," http://www-01.ibm.com/software/, last accessed 01.07.2021. 